\title{
Identificación de un modelo lineal adecuado para evaluar características pre-destete en ganado criollo colombiano Blanco Orejinegro
}

\author{
Identification of an appropriate linear model to evaluate pre-weaning traits in \\ colombian creole cattle Blanco Orejinegro
}

\author{
L. F. Naranjo-Guerrero ; A . López-Herrera iD , J. C. Rincón-Flórez iD , L. G. González-Herrera iD \\ DOI: https://doi.org/10.22517/23447214.24301 \\ Artículo de investigación científica y tecnológica
}

\begin{abstract}
The Blanco Orejinegro (BON) Creole breed has an adaptation process of more than $\mathbf{5 0 0}$ years to the environmental conditions of Colombia, and it is characterized by being a dualpurpose breed used for both milk and meat production, so it is a very important biological heritage that must be studied. The goal of this study was to identify an appropriate linear model to evaluate pre-weaning traits in $\mathrm{BON}$ cattle. Weighing information was collected and filtered from four BON cattle herds. The evaluated traits were weight at 4 months (W4M), weight at weaning (WW) and daily weight gain between 4 months and weaning (DWG4M-W). Nine linear models were evaluated in which were included the following factors as fixed effects: sex, herd, month of weighing or month of birth, calving number, time of weighing or birth (dry and rainy weather), age (covariate, fixed effect and adjusted by regression), year of weaning or year of birth and, contemporary group (CG) formed by sex and herd for DWG4M-W and sex, herd and year of weighing for W4M and WW, with a minimum of five observations in each CG. To identify the most appropriate linear model for each trait, the value of AIC (Akaike information criterion, BIC (Bayesian information criterion), determination coefficient $\left(R^{2}\right)$ and sum of squares of error (SCE) were used. The most suitable model for all the traits was the one that involved the contemporary group and age as a fixed effect for $\mathrm{W} 4 \mathrm{M}$ and age as a covariate for $\mathrm{WW}$.
\end{abstract}

Index Terms - growth, grouped age, contemporary group, weaning weigh

Resumen - La Raza criolla Blanco Orejinegro (BON) tiene un proceso de adaptación de más de 500 años a las condiciones ambientales de Colombia. Se caracteriza por ser una raza doble propósito utilizada para la producción de leche y carne, convirtiéndola en un patrimonio biológico de gran importancia que debe ser estudiado. El objetivo de este estudio fue identificar

This manuscript was sent on May 23, 2020 and accepted on February 05, 2021.

. L.F. Naranjo, Universidad Nacional de Colombia, Sede Medellín, (e-mail: lfnaranjog@unal.edu.co).

A. López-Herrera, Profesor titular, docente-investigador, dedicación Exclusiva. Director del Laboratorio de Biotecnología Animal, Departamento de Producción Animal, Facultad de Ciencias Agrarias, Universidad Nacional de Colombia, Sede Medellín, Calle 59 A \# 63-20, (e-mail: alherrera@unal.edu.co). un modelo lineal adecuado para evaluar características predestete en ganado criollo Blanco Orejinegro. Se recolectó y depuró información de pesajes de cuatro hatos de ganado BON. Las características evaluadas fueron peso a los 4 meses $(\mathrm{P} 4 \mathrm{M})$, peso al destete (PD) y ganancia diaria de peso entre los 4 meses y el destete (GDP4M-D). Se evaluaron nueve modelos lineales en los que se incluyeron como efectos fijos los siguientes factores: sexo, hato, mes de pesaje o nacimiento, número de parto, época de pesaje o época de nacimiento (época seca o lluviosa), edad (covariable, efecto fijo y ajustada por regresión), año de pesaje o año de nacimiento y grupo contemporáneo (GC) compuesto por sexo y hato para GDP4M-D y sexo, hato y año de pesaje para P4M y PD, con mínimo cinco observaciones por GC. Para identificar el modelo lineal más adecuado para cada característica se utilizó el valor de AIC (Akaike information criterion), BIC (Bayesian information criterion), coeficiente de determinación $\left(R^{2}\right)$ y la suma de cuadrados del error (SCE). El modelo más adecuado para todas las características fue aquel que involucró el GC y edad como efecto fijo para P4M y edad como covariable para PD.

Palabras claves - crecimiento, edad agrupada, grupo contemporáneo, peso al destete

\section{INTRODUCCIÓN}

A ganadería bovina tiene un origen diferente a América y

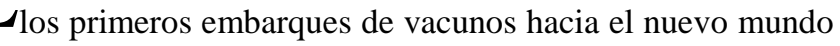
se dieron en el segundo viaje de Cristóbal Colón con destino hacia la isla de Santo Domingo, por lo cual todas las razas criollas colombianas son descendientes de las razas primitivas ibéricas traídas por los españoles [1]. Una de estas razas criollas es el Blanco Orejinegro (BON), la cual se caracteriza fenotípicamente por presentar pelaje de color blanco sobre piel

J. C. Rincón, Zoot, MSc, PhD. Profesor asociado. Departamento de Ciencia Animal. Facultad de Ciencias Agropecuarias. Universidad Nacional de Colombia, Sede Palmira, calle 32 \#12-00 (e-mail: jcrincon@unal.edu.co).

L.G González-Herrera, Docente investigador Asistente Dedicación Exclusiva, Departamento de Producción Animal, Facultad de Ciencias Agrarias, Universidad Nacional de Colombia, Sede Medellín, Calle59 A \# 6320 (e-mail: luggonzalezhe@unal.edu.co) 
negra en todo el cuerpo, orejas y morro negro. Los terneros nacen con piel rosada, pero a medida que van creciendo se va convirtiendo en negra por los estímulos del ambiente, especialmente la radiación solar; el proceso de pigmentación se completa alrededor de los 24 meses de edad [2], [3].

Existen características de interés económico relacionadas con el crecimiento pre-destete de los animales, dentro de las cuales se puede mencionar el peso a los 4 meses, el peso al destete y la ganancia diaria de peso entre los 4 meses y el destete. Estas características deben ser corregidas por algunos factores ambientales que se deben aplicar para todo el hato. Las correcciones a tener en cuenta están relacionadas con factores ambientales y factores propios del ternero. Para los factores ambientales se pueden mencionar la época, año y/o mes de nacimiento y de pesaje, edad y número de parto de la vaca, y dentro de los propios al ternero se encuentran el sexo, edad, su estado fisiológico, entre otras. [4]

El peso a los 4 meses es un importante indicador en la medición de la habilidad materna que puede ser evaluado en los terneros de forma temprana. La tasa de crecimiento de los terneros se mide generalmente por su comportamiento pre-destete a través de las ganancias diarias de peso, las cuales se ven afectadas por factores genéticos y ambientales que limitan el potencial de producción [5]. Por su parte, el peso al deteste refleja en gran medida la capacidad materna y habilidad que tiene una vaca para criar terneros, además de la capacidad propia del ternero de ganar peso [6]. Finalmente, el conocimiento de la influencia que tienen factores de tipo ambiental sobre el crecimiento de los animales es de suma importancia y por ende, se hace necesario encontrar modelos lineales que sean capaces de explicar de manera correcta la estructura y variabilidad de los datos. Por tal razón, el objetivo de este estudio fue identificar un modelo lineal adecuado para evaluar características predestete en ganado criollo colombiano Blanco Orejinegro (BON)

\section{Metodología}

El estudio se llevó a cabo con información productiva de cuatro hatos de ganado BON (identificados como A, B, C, D por confidencialidad), ubicados en los departamentos de Risaralda, Caldas y Antioquia.; Los hatos A y B se encuentran ubicados a una altura de 1000 m.s.n.m, temperatura promedio de $25^{\circ} \mathrm{C}$ y una humedad relativa de $76 \%$, los animales se alimentan de pasturas como Brachiaria toledo, Brachiaria decumbens, y Cynodon plectostachyus, con periodos de rotación que dependen del tamaño del potrero y con una recuperación de 35 días, el hato $\mathrm{C}$ está ubicada a una altura de 450 m.s.n.m, temperatura promedio de $28^{\circ} \mathrm{C}$, con una precipitación de 5085 $\mathrm{mm}$ de agua en el último año, posee 75 ha en pastoreo, compuestas por Brachiaria brizanta, Brachiaria decumbens, Brachiaria humidicola, Panicum máximum tanzania, Panicum máximum mombaza, Tifton 78 y grama; finalmente, el hato D se encuentra ubicado en una zona de vida de bosque húmedo montano bajo (bmh-MB), a una altura de 2500 m.s.n.m, temperatura promedio de $14^{\circ} \mathrm{C}$. Los animales son alimentados con pasto Kikuyo (Cenchrus clandestinum), con rotación de pastoreos en 14 ha
La información productiva se recolectó a partir de bases de datos físicas y electrónicas desde el año 2000 hasta el 2018, la cual fue digitalizada en el programa Excel y posteriormente fue depurada y analizada estadísticamente por medio del programa R Project versión 3.5.2 [7], eliminando datos fuera de los rangos biológicamente posibles o valores extremos. Las características de crecimiento pre-destete evaluadas fueron: peso a los 4 meses (P4M), peso al destete (PD) y ganancia diaria de peso entre los 4 meses y el destete (GDP4M-D). Según los parámetros fisiológicos de la raza, para $\mathrm{P} 4 \mathrm{M}$ se conservaron pesos entre 60 y $150 \mathrm{~kg}$, para el caso de PD se dejaron pesos entre 100 y 350 $\mathrm{kg}$; finalmente, para GDP4M-D se dejaron ganancias mayores a $0 \mathrm{~kg}$ y menores a $1 \mathrm{~kg}$. Los P4M y PD fueron modelados de acuerdo a tres diferentes criterios de edad al momento de estos pesajes, por haberse verificado este efecto como fuente de variación para estas características, inclusive, con resultados diferentes si se consideraba la edad como efecto fijo, como covariable o como edad ajustada. Las tres maneras como se incluyó la edad en el modelo, fueron: peso ajustado con base a la edad por regresión lineal simple, edad al momento del pesaje como covariable (con efecto lineal) y edad agrupada en cuatro categorías (efecto fijo) de la siguiente manera: Edad a los 4 meses en días (x): grupo 1: $90 \leq x \leq 100$, grupo 2: $100<x \leq 110$, grupo 3: $110<x \leq 125$, grupo 4: $125<x \leq 150$. Edad al destete en días: grupo 1: $180 \leq x \leq 225$, grupo $2: 225<x \leq 260$, grupo 3: $260<x \leq 275$, grupo 4: $275<x \leq 330$. De esta manera, para estas dos características, se utilizaron los mismos modelos, pero a la hora de incluir el efecto de edad, el modelado se hace de tres maneras diferentes. Se obtuvieron un total de 214 registros para P4M, 485 registros para PD y 175 registros para GDP4MD.

Para la evaluación de los modelos se tuvieron en cuenta como efectos fijos; sexo, hato, mes de pesaje, mes de nacimiento, número de parto, época de pesaje, época de nacimiento, edad (incluyendo los tres criterios propuestos para este efecto), año de pesaje o de nacimiento y grupo contemporáneo (GC) compuesto por la concatenación de sexo y hato, para GDP4M$\mathrm{D}$, y por sexo, hato y año de pesaje, para P4M y PD. Para que un GC permaneciera en los análisis, debía contar con mínimo cinco observaciones para las tres características de interés. El P4M y PD consideraron dentro de los modelos; el mes, año y época de pesaje, y la GDP4M-D consideró dentro del modelo el mes, año y época de nacimiento. La época de nacimiento y la época de pesaje fue formada con base a los comportamientos reportados en las zonas evaluadas, obteniéndose, dos épocas secas, la primera comprendida por los meses de enero, febrero y marzo y la segunda comprendida por julio, agosto y septiembre; de igual manera, dos épocas lluviosas, la primera comprendida por los meses de abril, mayo y junio, y la segunda comprendida por octubre, noviembre y diciembre. Para el número de parto, se agruparon partos iguales y mayores a 7 , debido al bajo número de observaciones que se tenía para estas órdenes de parto superiores; además, se comprobó gráficamente que la media de los pesos después del parto 6 tenía una tendencia a disminuir levemente, por lo que al agrupar el número de parto 7 o más, se considera el efecto cuadrático que tiene el número de parto sobre los pesajes de los terneros. 
Con base en lo descrito anteriormente, se evaluaron nueve modelos para cada una de las características (Tablas I, II y III) y para cada uno de los criterios modelados (edad agrupada, edad ajustada y edad como covariable) para las características P4M y PD, iniciando con el modelo más simple, el cual incluía únicamente uno de los efectos y se iban agregando paulatinamente los otros efectos anteriormente mencionados, para completar el resto de los modelos evaluados. En (1) se describe un modelo general incluyendo todas las fuentes de variación.

$\mathrm{Y}_{\mathrm{IJKLMNOP}}=\mu+\mathrm{H}_{\mathrm{I}}+\mathrm{S}_{\mathrm{J}}+\mathrm{EP}_{\mathrm{K}+} \mathrm{MP}_{\mathrm{L}}+\mathrm{AP}_{\mathrm{M}}+\mathrm{NP}_{\mathrm{N}}+\mathrm{GC}_{\mathrm{O}}+\mathrm{ED}_{\mathrm{P}}+\mathrm{E}_{\text {IJKLMNOP }}$

Donde,

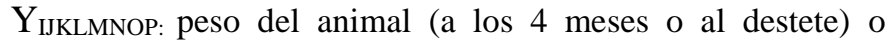
ganancia de peso entre los 4 meses y el destete, en el I-ésimo hato, del J-ésimo sexo, en la K-ésima época de nacimiento o pesaje, L-ésimo mes de nacimiento o pesaje, M-ésimo año de nacimiento o pesaje, $\mathrm{N}$-ésimo número de parto, perteneciente al O-ésimo grupo contemporáneo y con una P-ésima edad a los 4 meses y al destete.

$\mu$ : media general

$\mathrm{H}_{\mathrm{I}}$ : efecto del I-ésimo hato (4 hatos)

SJ: efecto del J-ésimo sexo (macho o hembra)

$\mathrm{EP}_{\mathrm{K}}$ : efecto de la K-ésima época de nacimiento (ganancia diaria de peso entre los 4 meses y el destete) o pesaje (pesos a los 4 meses y al destete) $(\mathrm{K}=1,2,3,4)$

$\mathrm{MP}_{\mathrm{L}}$ : efecto del L-ésimo mes de nacimiento (ganancia diaria de peso entre los 4 meses y el destete) o pesaje (peso a los 4 meses y el destete) $(\mathrm{L}=1,2, \ldots, 12)$

$\mathrm{AP}_{\mathrm{M}}$ : efecto del M-ésimo año de nacimiento (ganancia diaria de peso entre los 4 meses y el destete) o pesaje (peso a los 4 meses y el destete $)(M=2000, \ldots, 2018)$

$\mathrm{NP}_{\mathrm{N}}$ : efecto del $\mathrm{N}$-ésimo número de parto $(\mathrm{N}=1,2, \ldots, 7$ o más $)$ $\mathrm{GC}_{\mathrm{o}}$ : efecto del O-ésimo grupo contemporáneo compuesto por hato y sexo para ganancia de peso entre los 4 meses y el destete y hato, sexo y año de pesaje para peso a los 4 meses y peso al destete $(\mathrm{O}=1,2, \ldots, \mathrm{O})$

EDp: efecto de la P-ésima edad agrupada, como covariable o ajustada, para las características peso a los 4 meses $(\mathrm{P} 4 \mathrm{M})$ y peso al destete (PD)

EIJKLMNOP: error aleatorio asociado a cada observación

Se llevó a cabo en el programa $\mathrm{R}$ versión 3.5.2 [6] una comparación de los modelos por medio de análisis de varianza tipo III, considerando que los datos se encontraban desbalanceados, para determinar el modelo más adecuado en la descripción de la variabilidad de los datos y se usaron como criterios de comparación de modelos, los valores de AIC (Akaike information criterion) y BIC (Bayesian information criterion), coeficiente de determinación $\left(\mathrm{R}^{2}\right)$ y suma de cuadrados del error (SCE); seleccionando los modelos con valores de AIC y BIC más cercanos a cero, mayores $\mathrm{R}^{2}$ y menores SCE. Previo a los análisis mencionados, se comprobó la existencia de los supuestos de normalidad y homocedasticidad de los residuos por medio de la prueba de Shapiro Wilk y la prueba de Levene, respectivamente.

\section{RESULTADOS Y DISCUSIONES}

La media para la característica P4M para los ejemplares de la raza $\mathrm{BON}$ fue de $110.68 \pm 18.84 \mathrm{~kg}$ con un coeficiente de variación $(\mathrm{CV})$ de $17.02 \%$. Las comparaciones para esta característica se realizan con otras razas, debido a que para $\mathrm{P} 4 \mathrm{M}$ en la raza $\mathrm{BON}$ no se tiene información disponible. Estos resultados son inferiores a los pesos promedios reportados en varios estudios en Brasil para la raza Nelore con valores de $121.0 \pm 21.1 \mathrm{~kg}$ [8], $134 \mathrm{~kg}$ [9], $127.51 \pm 16.90 \mathrm{~kg}$ [10] у 129 $\mathrm{kg}$ [11] y similares a los promedios reportados en un estudio realizado en Argentina, para evaluar cruces de la raza Senepol y de la raza Angus con valores de $111.15 \pm 14.34 \mathrm{~kg}$ y 117.61 $\pm 12.85 \mathrm{~kg}$, respectivamente [12].

La superioridad encontrada para la raza Nelore (Bos indicus) evaluada en Brasil comparada con la raza BON (Bos taurus), puede deberse a que la primera es una raza de mayor tamaño y por ende con mayores pesos; además, en la raza Nelore, que es especializada en producción de carne, se han realizado programas de selección durante muchos años con base en valores genéticos, obteniéndose animales con mayor potencial genético para dicha característica, situación que no se ha presentado en la raza BON para P4M, pero, que a pesar de ello, muestra un buen desempeño para esta característica, demostrando así el potencial que puede llegar a tener la raza BON, si se hace selección de animales superiores, con base en el valor genético para P4M. De esta manera, la evaluación genética de características de crecimiento, como el peso corporal y medidas en la fase inicial de desarrollo de los animales, toma importancia en la determinación de la eficiencia económica en cualquier sistema de producción y pueden ser utilizadas como criterios de selección [8].

Para la característica PD en los BON de este trabajo se encontró una media de $203.95 \pm 30.72 \mathrm{~kg}$ con un CV de $17.03 \%$ y una media de edad de $261.39 \pm 30.49$ días, con un CV de $11.67 \%$. Estos resultados son superiores a los reportados por estudios realizados en la raza $\mathrm{BON}$ con valores medios de $190.29 \pm 5.50$ $\mathrm{kg}$ [13], $165 \pm 29.16 \mathrm{~kg}$ para PD ajustado a los 240 días de edad [14], $196.3 \pm 31.4 \mathrm{~kg}$ a una edad promedio de $271.8 \pm 13.5$ días [15] y un PD ajustado a 240 días de $169 \pm 24.4 \mathrm{~kg}$ [16]. Aunque los PD en cada uno de los estudios fueron evaluados a diferentes edades, se puede ver la superioridad del BON, el cual presentó mayor media de PD en un menor tiempo; esta superioridad puede ser el reflejo de las mejoras que se han realizado para esta raza en algunos de los hatos estudiados en cuanto a manejo, alimentación, condiciones sanitarias y formas de selección para PD.

Esta característica toma importancia debido a que muestra el comportamiento pre-destete que ha tenido el ternero, en una etapa de alta tasa de crecimiento, donde el animal a los ocho meses ha alcanzado entre el 25 al $42 \%$ del peso final, siendo esta una fase en donde podrían seleccionarse animales para peso al destete contribuyendo a la selección de machos y hembras a través de los valores genéticos obtenidos; además, refleja en gran medida la capacidad materna y habilidad que tiene una vaca para criar terneros [6] , [17] 
Para la característica GDP4M-D tampoco se tiene información en la raza BON. En el presente estudio, se encontró un valor promedio de $0.625 \pm 0.162 \mathrm{~kg}$ con un $\mathrm{CV}$ de $25.98 \%$. En estudios realizados en la raza Nelore, se encontró una ganancia de peso total entre los 120 y los 240 días de edad de $66.94 \mathrm{~kg}$, es decir $0.56 \mathrm{~kg}$ por día [18]. Debido a que las ganancias de peso fueron evaluadas en periodos diferentes en ambos estudios, no se puede hacer una comparación directa entre estas medias; sin embargo, se puede ver la capacidad que tiene la raza BON para obtener buenas ganancias de peso, conociendo que en esta raza el mejoramiento genético que se ha hecho en Colombia a partir de evaluaciones genéticas es casi nulo, además, que son animales que no se suplementan y están bajo sistemas de pastoreo.

En la tabla I y II, se reportan los valores de criterios de selección de 11 modelos evaluados para las características P4M y PD, evaluando la edad como covariable, edad ajustada por regresión y edad agrupada, respectivamente. En la tabla III, se reportan los valores de criterios de selección para GDP4M-D. En negrilla en cada tabla se resaltan los modelos que mejores valores presentaron, de acuerdo con los criterios de comparación entre modelos y se diferencia además con letra cursiva y negrilla el modelo que fue seleccionado.

TABLA I

CRITERIOS DE SELECCIÓN PARA LA CARACTERÍSTICA PESO A LOS 4 MESES.

\begin{tabular}{|c|c|c|c|c|c|c|}
\hline & & Modelo & AIC & $\mathrm{BIC}$ & $\mathrm{R} 2$ & SCE \\
\hline 1 & & $\mu+\mathrm{H}_{I}+\mathrm{E}_{\mathrm{I}}$ & 1940 & 1957 & 0.03 & 74780 \\
\hline 2 & & $\mu+\mathrm{H}_{\mathrm{I}}+\mathrm{S}_{\mathrm{J}}+\mathrm{E}_{\mathrm{IJ}}$ & 1941 & 1962 & 0.03 & 74695 \\
\hline 3 & $\begin{array}{l}E \\
D\end{array}$ & $\mu+\mathrm{H}_{\mathrm{I}}+\mathrm{S}_{\mathrm{J}}+\mathrm{EP}_{\mathrm{K}}+\mathrm{E}_{\mathrm{JK}}$ & 1937 & 1967 & 0.08 & 71162 \\
\hline 4 & D & $\mu+\mathrm{H}_{\mathrm{I}}+\mathrm{S}_{\mathrm{J}}+\mathrm{MP}_{\mathrm{L}}+\mathrm{E}_{\mathrm{JJL}}$ & 1947 & 2005 & 0.10 & 69437 \\
\hline 5 & $\mathrm{~A}$ & $\mu+\mathrm{H}_{I}+\mathrm{S}_{J}+\mathrm{AP}_{M}+\mathrm{E}_{\text {JKM }}$ & 1917 & 1971 & 0.21 & 61138 \\
\hline 6 & C & $\mu+\mathrm{H}_{I}+\mathrm{S}_{J}+\mathrm{AP}_{\mathrm{M}}+\mathrm{NP}_{\mathrm{N}}+\mathrm{E}_{\text {IJMN }}$ & 1910 & 1985 & 0.27 & 56444 \\
\hline 7 & $\mathrm{O}$ & $\mu+\mathrm{H}_{\mathrm{I}}+\mathrm{S}_{\mathrm{J}}+\mathrm{EP}_{\mathrm{K}}+\mathrm{NP}_{\mathrm{N}}+\mathrm{ED}_{\mathrm{P}}+\mathrm{E}_{\mathrm{IJKNP}}$ & 1870 & 1924 & 0.36 & 49552 \\
\hline 8 & & $\mu+\mathrm{NP}_{\mathrm{N}}+\mathrm{GC}_{\mathrm{O}}+\mathrm{ED}_{\mathrm{P}}+\mathrm{E}_{\mathrm{NOP}}$ & 1820 & 1947 & 0.57 & 32942 \\
\hline 9 & & $\mu+\mathrm{H}_{\mathrm{I}}+\mathrm{EP}_{\mathrm{K}}+\mathrm{MP}_{\mathrm{L}}+\mathrm{AP}_{\mathrm{M}}+\mathrm{NP}_{\mathrm{N}}+\mathrm{ED}_{\mathrm{P}}+\mathrm{E}_{\mathrm{IKLMNP}}$ & 1850 & 1963 & 0.49 & 39063 \\
\hline 1 & & $\mu+\mathrm{H}_{\mathrm{I}}+\mathrm{E}_{\mathrm{I}}$ & 1893 & 1910 & 0.04 & 60774 \\
\hline 2 & & $\mu+\mathrm{H}_{\mathrm{I}}+\mathrm{S}_{\mathrm{J}}+\mathrm{E}_{\mathrm{IJ}}$ & 1895 & 1916 & 0.04 & 60748 \\
\hline 3 & & $\mu+\mathrm{H}_{I}+\mathrm{S}_{J}+\mathrm{EP}_{\mathrm{K}}+\mathrm{E}_{\mathrm{JJK}}$ & 1890 & 1920 & 0.09 & 57711 \\
\hline 4 & $\mathrm{E}$ & $\mu+\mathrm{H}_{\mathrm{I}}+\mathrm{S}_{\mathrm{J}}+\mathrm{MP}_{\mathrm{L}}+\mathrm{E}_{\mathrm{IJL}}$ & 1899 & 1957 & 0.12 & 56098 \\
\hline 5 & $\mathrm{D}$ & $\mu+\mathrm{H}_{I}+\mathrm{S}_{\mathrm{J}}+\mathrm{AP}_{\mathrm{M}}+\mathrm{E}_{\mathrm{IJM}}$ & 1851 & 1906 & 0.28 & 45650 \\
\hline 6 & $\mathrm{~A}$ & $\mu+\mathrm{H}_{I}+\mathrm{S}_{\mathrm{J}}+\mathrm{AP}_{\mathrm{M}}+\mathrm{NP}_{\mathrm{N}}+\mathrm{E}_{\text {IJMN }}$ & 1843 & 1918 & 0.34 & 41595 \\
\hline 7 & & $\mu+\mathrm{H}_{I}+\mathrm{S}_{J}+\mathrm{EP}_{\mathrm{K}}+\mathrm{NP}_{\mathrm{N}}+\mathrm{E}_{\mathrm{IJKN}}$ & 1873 & 1924 & 0.20 & 50673 \\
\hline 8 & & $\mu+\mathrm{NP}_{\mathrm{N}}+\mathrm{GC}_{\mathrm{O}}+\mathrm{E}_{\mathrm{NO}}$ & 1828 & 1950 & 0.46 & 34324 \\
\hline 9 & & $\mu+\mathrm{H}_{\mathrm{I}}+\mathrm{EP}_{\mathrm{K}}+\mathrm{MP}_{\mathrm{L}}+\mathrm{AP}_{\mathrm{M}}+\mathrm{NP}_{\mathrm{N}}+\mathrm{E}_{\text {IKLMN }}$ & 1857 & 1966 & 0.36 & 46726 \\
\hline 1 & & $\mu+\mathrm{H}_{\mathrm{I}}+\mathrm{E}_{\mathrm{I}}$ & 1940 & 1957 & 0.03 & 74780 \\
\hline 2 & & $\mu+\mathrm{H}_{I}+\mathrm{S}_{\mathrm{J}}+\mathrm{E}_{\mathrm{IJ}}$ & 1941 & 1962 & 0.03 & 74695 \\
\hline 3 & & $\mu+\mathrm{H}_{I}+\mathrm{S}_{\mathrm{J}}+\mathrm{EP}_{\mathrm{K}}+\mathrm{E}_{\mathrm{JJK}}$ & 1937 & 1967 & 0.08 & 71162 \\
\hline 4 & E & $\mu+\mathrm{H}_{\mathrm{I}}+\mathrm{S}_{\mathrm{J}}+\mathrm{MP}_{\mathrm{L}}+\mathrm{E}_{\mathrm{IJL}}$ & 1947 & 2005 & 0.10 & 69437 \\
\hline 5 & $\mathrm{D}$ & $\mu+\mathrm{H}_{I}+\mathrm{S}_{J}+\mathrm{AP}_{\mathrm{M}}+\mathrm{E}_{\mathrm{IJM}}$ & 1917 & 1971 & 0.21 & 61138 \\
\hline 6 & $\mathrm{~A}$ & $\mu+\mathrm{H}_{I}+\mathrm{S}_{\mathrm{J}}+\mathrm{AP}_{\mathrm{M}}+\mathrm{NP}_{\mathrm{N}}+\mathrm{E}_{\text {IJMN }}$ & 1911 & 1985 & 0.27 & 56444 \\
\hline 7 & G & $\mu+\mathrm{H}_{\mathrm{I}}+\mathrm{S}_{\mathrm{J}}+\mathrm{EP}_{\mathrm{K}}+\mathrm{NP}_{\mathrm{N}}+\mathrm{ED}_{\mathrm{P}}+\mathrm{E}_{\mathrm{IJKNP}}$ & 1881 & 1942 & 0.34 & 51129 \\
\hline 8 & & $\mu+\mathrm{NP}_{\mathrm{N}}+\mathrm{GC}_{\mathrm{O}}+\mathrm{ED}_{\mathrm{P}}+\mathrm{E}_{\mathrm{NOP}}$ & $\underline{1822}$ & 1955 & $\underline{0.58}$ & $\underline{32558}$ \\
\hline 9 & & $\mu+\mathrm{H}_{I}+\mathrm{EP}_{\mathrm{K}}+\mathrm{MP}_{\mathrm{L}}+\mathrm{AP}_{\mathrm{M}}+\mathrm{NP}_{\mathrm{N}}+\mathrm{ED}_{\mathrm{P}}+\mathrm{E}_{I K L M N}$ & 1858 & 1955 & 0.49 & 39637 \\
\hline
\end{tabular}

Para P4M y PD (Tabla I y II) al comparar los valores de BIC en los criterios de edad como covariable (EDACOV) y edad agrupada (EDAG), se puede observar que el modelo con menor valor de BIC fue el 7 en ambas características, mientras que para el caso del criterio de edad ajustada (EDA), el modelo que menor valor de BIC presentó en ambas características fue el 5. Sin embargo, el modelo seleccionado fue el modelo 8 con edad agrupada (EDAG) para P4M, y con edad como covariable (EDACOV) para PD. Este modelo fue el que involucró el grupo contemporáneo, mostrando menores valores de AIC y suma de cuadrados del error (SCE) y mayores de $\mathrm{R}^{2}$ con valores de BIC no muy cercanos a cero. Esta situación puede darse debido a que este último criterio penaliza aquellos modelos con mayor número de parámetros, por lo cual, tiende a seleccionar los modelos más simples [19]. En este caso, la inclusión del grupo contemporáneo (hato, sexo, año de pesaje), implica un mayor número de estimativas, debido a la interacción que se genera entre los efectos aquí involucrados.

TABLA II

CRITERIOS DE SELECCIÓN PARA LA CARACTERÍSTICA PESO AL DESTETE.

\begin{tabular}{|c|c|c|c|c|c|c|}
\hline & & Modelo & $\mathrm{AIC}$ & $\mathrm{BIC}$ & $\mathrm{R} 2$ & SCE \\
\hline 1 & & $\mu+\mathrm{H}_{\mathrm{I}}+\mathrm{E}_{\mathrm{J}}$ & 4900 & 4922 & 0.15 & 527389 \\
\hline 2 & $\mathrm{E}$ & $\mu+\mathrm{H}_{\mathrm{I}}+\mathrm{S}_{\mathrm{J}}+\mathrm{E}_{\mathrm{IJ}}$ & 4876 & 4902 & 0.19 & 500323 \\
\hline 3 & $\mathrm{D}$ & $\mu+\mathrm{H}_{\mathrm{I}}+\mathrm{S}_{\mathrm{J}}+\mathrm{EP}_{\mathrm{K}}+\mathrm{E}_{\mathrm{JK}}$ & 4870 & 4908 & 0.21 & 487955 \\
\hline 4 & A & $\mu+\mathrm{H}_{\mathrm{I}}+\mathrm{S}_{\mathrm{J}}+\mathrm{MP}_{\mathrm{L}}+\mathrm{E}_{\mathrm{JLL}}$ & 4857 & 4929 & 0.25 & 460865 \\
\hline 5 & $\mathrm{D}$ & $\mu+\mathrm{H}_{\mathrm{I}}+\mathrm{S}_{\mathrm{J}}+\mathrm{AP}_{\mathrm{M}}+\mathrm{E}_{\mathrm{IJKM}}$ & 4836 & 4925 & 0.30 & 434721 \\
\hline 6 & $\mathrm{C}$ & $\mu+\mathrm{H}_{I}+\mathrm{S}_{\mathrm{J}}+\mathrm{AP}_{\mathrm{M}}+\mathrm{NP}_{\mathrm{N}}+\mathrm{E}_{\text {IJMN }}$ & 4822 & 4936 & 0.33 & 412714 \\
\hline 7 & $\mathrm{O}$ & $\mu+\mathrm{H}_{\mathrm{I}}+\mathrm{S}_{\mathrm{J}}+\mathrm{EP}_{\mathrm{K}}+\mathrm{NP}_{\mathrm{N}}+\mathrm{EDP}_{\mathrm{P}}+\mathrm{E}_{\mathrm{IJKNP}}$ & 4803 & 4871 & 0.33 & 415153 \\
\hline 8 & $\mathrm{~V}$ & $\mu+\mathrm{EP}_{\mathrm{K}}+\mathrm{NP}_{\mathrm{N}}+\mathrm{GC}_{\mathrm{O}}+\mathrm{ED}_{\mathrm{P}}+\mathrm{E}_{\mathrm{KNOP}}$ & $\underline{4619}$ & 4893 & $\underline{0.62}$ & $\underline{235820}$ \\
\hline 9 & & $\mu+\mathrm{H}_{\mathrm{I}}+\mathrm{EP}_{\mathrm{K}}+\mathrm{MP}_{\mathrm{L}}+\mathrm{AP}_{\mathrm{M}}+\mathrm{NP}_{\mathrm{N}}+\mathrm{ED}_{\mathrm{P}}+\mathrm{E}_{\text {IKLMNP }}$ & $\overline{4812}$ & 4972 & $\overline{0.37}$ & $\overline{386908}$ \\
\hline 1 & & $\mu+\mathrm{H}_{\mathrm{I}}+\mathrm{E}_{\mathrm{I}}$ & 4891 & 4912 & 0.15 & 491242 \\
\hline 2 & & $\mu+\mathrm{H}_{\mathrm{I}}+\mathrm{S}_{\mathrm{J}}+\mathrm{E}_{\mathrm{IJ}}$ & 4869 & 4894 & 0.19 & 467862 \\
\hline 3 & & $\mu+\mathrm{H}_{\mathrm{I}}+\mathrm{S}_{\mathrm{J}}+\mathrm{EP}_{\mathrm{K}}+\mathrm{E}_{\mathrm{JK}}$ & 4856 & 4895 & 0.22 & 451105 \\
\hline 4 & $\mathrm{E}$ & $\mu+\mathrm{H}_{\mathrm{I}}+\mathrm{S}_{\mathrm{J}}+\mathrm{MP}_{\mathrm{L}}+\mathrm{E}_{\mathrm{JJL}}$ & 4834 & 4907 & 0.28 & 418426 \\
\hline 5 & $\mathrm{D}$ & $\mu+\mathrm{H}_{I}+\mathrm{S}_{\mathrm{J}}+\mathrm{AP}_{\mathrm{M}}+\mathrm{E}_{I J M}$ & 4790 & 4878 & 0.35 & 376533 \\
\hline 6 & A & $\mu+\mathrm{H}_{I}+\mathrm{S}_{\mathrm{J}}+\mathrm{AP}_{\mathrm{M}}+\mathrm{NP}_{\mathrm{N}}+\mathrm{E}_{\mathrm{IJMN}}$ & 4768 & 4882 & 0.39 & 352031 \\
\hline 7 & & $\mu+\mathrm{H}_{I}+\mathrm{S}_{\mathrm{J}}+\mathrm{EP}_{K}+\mathrm{NP}_{\mathrm{N}}+\mathrm{E}_{\mathrm{JKN}}$ & 4833 & 4897 & 0.27 & 420774 \\
\hline 8 & & $\mu+\mathrm{EP}_{\mathrm{K}}+\mathrm{NP}_{\mathrm{N}}+\mathrm{GC}_{\mathrm{O}}+\mathrm{E}_{\mathrm{KNO}}$ & 4646 & 4916 & 0.59 & 238272 \\
\hline 9 & & $\mu+\mathrm{H}_{\mathrm{I}}+\mathrm{EP}_{\mathrm{K}}+\mathrm{MP}_{\mathrm{L}}+\mathrm{AP}_{\mathrm{M}}+\mathrm{NP}_{\mathrm{N}}+\mathrm{E}_{\mathrm{IKLMN}}$ & 4817 & 4973 & 0.36 & 372842 \\
\hline 1 & & $\mu+\mathrm{H}_{\mathrm{I}}+\mathrm{E}_{\mathrm{I}}$ & 4900 & 4921 & 0.14 & 527389 \\
\hline 2 & & $\mu+\mathrm{H}_{\mathrm{I}}+\mathrm{S}_{\mathrm{J}}+\mathrm{E}_{\mathrm{IJ}}$ & 4876 & 4901 & 0.19 & 500323 \\
\hline 3 & $\mathrm{E}$ & $\mu+\mathrm{H}_{\mathrm{I}}+\mathrm{S}_{\mathrm{J}}+\mathrm{EP}_{\mathrm{K}}+\mathrm{E}_{\mathrm{JK}}$ & 4870 & 4907 & 0.21 & 487954 \\
\hline 4 & $\mathrm{D}$ & $\mu+\mathrm{H}_{\mathrm{I}}+\mathrm{S}_{\mathrm{J}}+\mathrm{MP}_{\mathrm{L}}+\mathrm{E}_{\mathrm{JL}}$ & 4857 & 4929 & 0.25 & 460865 \\
\hline 5 & A & $\mu+\mathrm{H}_{I}+\mathrm{S}_{\mathrm{J}}+\mathrm{AP}_{\mathrm{M}}+\mathrm{E}_{\mathrm{IJM}}$ & 4836 & 4924 & 0.30 & 434721 \\
\hline 6 & G & $\mu+\mathrm{HI}+\mathrm{SJ}+\mathrm{AP}_{\mathrm{M}}+\mathrm{NP}_{\mathrm{N}}+\mathrm{E}_{\mathrm{IJMN}}$ & 4822 & 4936 & 0.33 & 412714 \\
\hline 7 & & $\mu+\mathrm{H}_{\mathrm{I}}+\mathrm{S}_{\mathrm{J}}+\mathrm{EP}_{\mathrm{k}}+\mathrm{NP}_{\mathrm{N}}+\mathrm{EDP}_{\mathrm{P}}$ & 4814 & 4890 & 0.32 & 421251 \\
\hline 8 & & $\mu+\mathrm{EP}_{\mathrm{K}}+\mathrm{NP}_{\mathrm{N}}+\mathrm{GC}_{\mathrm{O}}+\mathrm{ED}_{\mathrm{P}}+\mathrm{E}_{\mathrm{KNOP}}$ & 4644 & 4927 & 0.60 & 246172 \\
\hline 9 & & $\mu+\mathrm{H}_{\mathrm{I}}+\mathrm{EP}_{\mathrm{K}}+\mathrm{MP}_{\mathrm{L}}+\mathrm{AP}_{\mathrm{M}}+\mathrm{NP}_{\mathrm{N}}+\mathrm{ED}_{\mathrm{P}}+\mathrm{E}_{\mathrm{IKLMP}}$ & 4826 & 4994 & 0.36 & 394671 \\
\hline
\end{tabular}

TABLA III

CRITERIOS DE SELECCIÓN PARA LA CARACTERÍSTICA GANANCIA DIARIA DE PESO ENTRE LOS 4 MESES Y EL DESTETE.

\begin{tabular}{llrrrr}
\hline \hline \multicolumn{1}{c}{ Modelo } & AIC & \multicolumn{1}{c}{ BIC } & \multicolumn{1}{c}{ R2 } & \multicolumn{1}{c}{ SCE } \\
\hline 1 & $\mu+\mathrm{H}_{I}+\mathrm{E}_{\mathrm{I}}$ & $\mathbf{- 1 5 4}$ & -138 & 0.001 & 4.19 \\
2 & $\mu+\mathrm{H}_{\mathrm{I}}+\mathrm{S}_{\mathrm{J}}+\mathrm{E}_{\mathrm{IJ}}$ & -158 & -139 & 0.04 & 4.04 \\
3 & $\mu+\mathrm{H}_{\mathrm{I}}+\mathrm{S}_{\mathrm{J}}+\mathrm{EP}_{\mathrm{K}}+\mathrm{E}_{\text {IJK }}$ & -166 & -138 & 0.11 & 3.74 \\
4 & $\mu+\mathrm{H}_{\mathrm{I}}+\mathrm{S}_{\mathrm{J}}+\mathrm{MP}_{\mathrm{L}}+\mathrm{E}_{\text {IJL }}$ & -156 & -102 & 0.13 & 3.63 \\
5 & $\mu+\mathrm{H}_{\mathrm{I}}+\mathrm{S}_{\mathrm{J}}+\mathrm{AP}_{\mathrm{M}}+\mathrm{E}_{\text {IJM }}$ & -170 & -112 & 0.21 & 3.32 \\
6 & $\mu+\mathrm{H}_{\mathrm{I}}+\mathrm{S}_{\mathrm{J}}+\mathrm{AP}_{\mathrm{M}}+\mathrm{NP}_{\mathrm{N}}+\mathrm{E}_{\text {IJMN }}$ & -165 & -89 & 0.24 & 3.18 \\
7 & $\mu+\mathrm{H}_{\mathrm{I}}+\mathrm{S}_{\mathrm{J}}+\mathrm{EP}_{\mathrm{k}}+\mathrm{NP}_{\mathrm{n}}+\mathrm{E}_{\text {IJKN }}$ & -158 & -110 & 0.13 & 3.66 \\
8 & $\mu+\mathrm{EP}_{\mathrm{K}}+\mathrm{AP}_{\mathrm{M}}+\mathrm{NP}_{\mathrm{n}}+\mathrm{GC}_{\mathrm{o}}+\mathrm{E}_{\mathrm{KMNO}}$ & -171 & -76 & $\underline{\mathbf{0 . 3 1}}$ & $\underline{\mathbf{2 . 8 7}}$ \\
9 & $\mu+\mathrm{H}_{\mathrm{I}}+\mathrm{EP}_{\mathrm{K}}+\mathrm{MP}_{\mathrm{L}}+\mathrm{AP}_{\mathrm{M}}+\mathrm{NP}_{\mathrm{N}}+\mathrm{E}_{\text {IKMLN }}$ & -157 & $\mathbf{- 4 5}$ & 0.29 & $\mathbf{2 . 9 4}$ \\
\hline \hline
\end{tabular}

Para GDP4M-D (Tabla III) el mejor modelo fue el número 8, que involucró también el grupo contemporáneo y que presentó mayor valor de $\mathrm{R}^{2}$ y menor suma de cuadrados del error. Sin embargo, la mejora en la descripción de la variabilidad de los datos explicada por un mayor $\mathrm{R}^{2}$ y menor suma de cuadrados del error, no es tan evidente como si lo es para las otras dos características. Adicional a esto, los valores de AIC y BIC no fueron los más cercanos a cero. Aunque BIC presentó menor valor que AIC, esto pudo deberse a que, en este modelo, el año de nacimiento fue analizado por fuera del grupo contemporáneo, lo que implica menos interacciones en el grupo contemporáneo (hato, sexo) y por tanto un modelo más sencillo. 
En general hay una tendencia al aumento del $\mathrm{R}^{2}$ y a la disminución de la suma de cuadrados del error, a medida que se incluyen nuevas fuentes de variación en los modelos, inclusive por el hecho de considerar interacción entre algunos factores, como ocurre con el uso del GC. Esto se puede deber en parte a que a medida que aumenta el número de efectos a evaluar se disminuyen los grados de libertad del error y por ende la suma de cuadrados del error, lo que refleja mayores valores de $\mathrm{R}^{2}$. Por otro lado, la inclusión del GC en el modelo para el P4M y PD explica casi el doble de la variabilidad encontrada en el modelo y disminuye a la mitad la imprecisión de las estimativas, según los valores de la suma de cuadrados del error. Estudios realizados acerca de los errores que se cometen al interpretar el $\mathrm{R}^{2}$ demuestran que este valor no está afectado únicamente por el tamaño muestral con el que se está trabajando, sino que está afectado por el número de variables explicativas consideradas en el modelo; esto se debe a que el $\mathrm{R}^{2}$ es una función no decreciente del número de variables presentes en el modelo, lo que implica que a mayor número de variables regresoras, mayor $\mathrm{R}^{2}$ [20]. De tal manera, la importancia de trabajar con modelos que involucran grupos contemporáneos para llevar a cabo evaluaciones genéticas en características de importancia económica radica en que se da una reducción de sesgos debidas a diferencias en el manejo y condiciones ambientales de los animales [15].

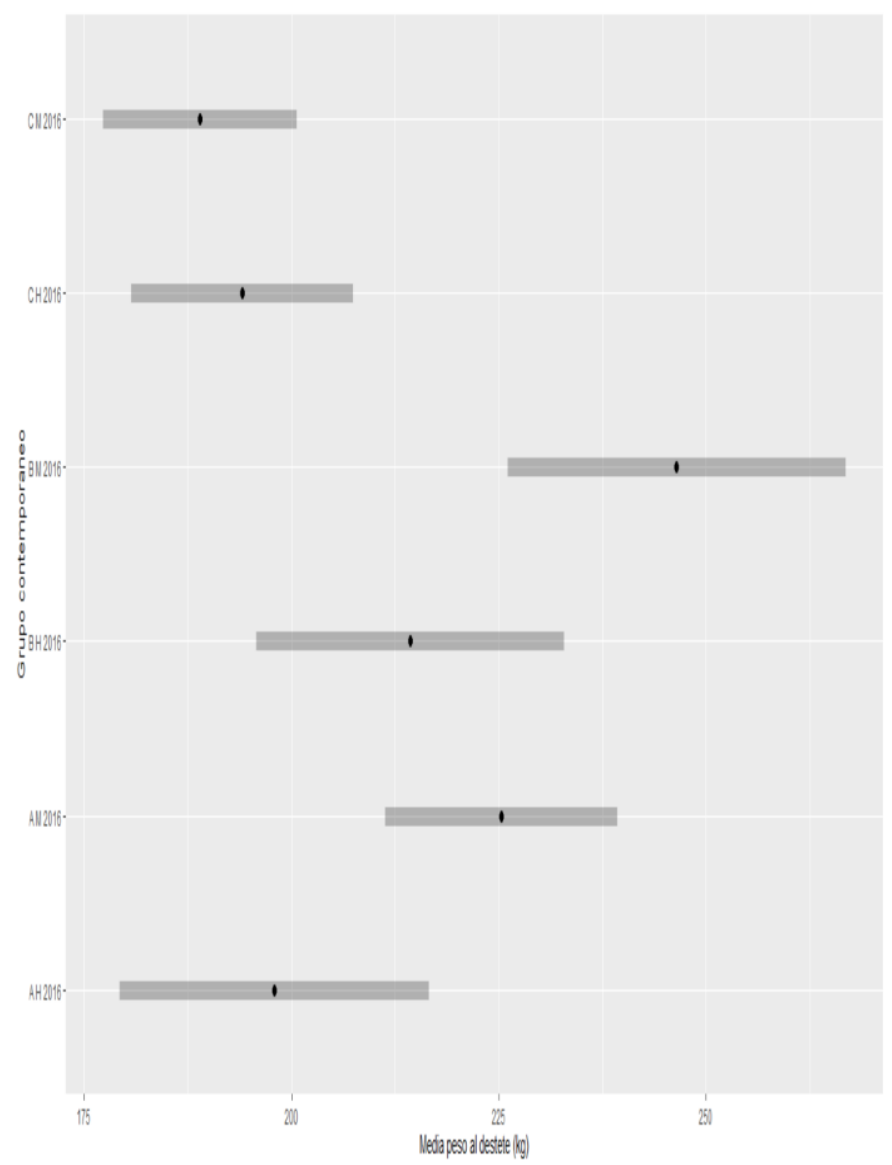

Fig.1. Interacción de hato y sexo para el año 2016.

En la Fig. 1 y 2 se observa un ejemplo de la interacción existente entre los diferentes efectos que conforman el grupo contemporáneo, por medio de un gráfico de medias ajustadas para la característica peso al destete. Para la Fig. 1 se evalúa la interacción entre hato y sexo durante el año 2016. Se puede observar que al evaluar las hembras y machos de la finca C, el grupo contemporáneo $\mathrm{CH} 2016$ (hembras pesadas en el año de 2016 en el hato C) muestra una mayor media para la característica PD, contrario a lo que se muestra en el resto de los GC en los que los machos de los otros hatos son quienes presentan mayor media para peso al destete en el año 2016.

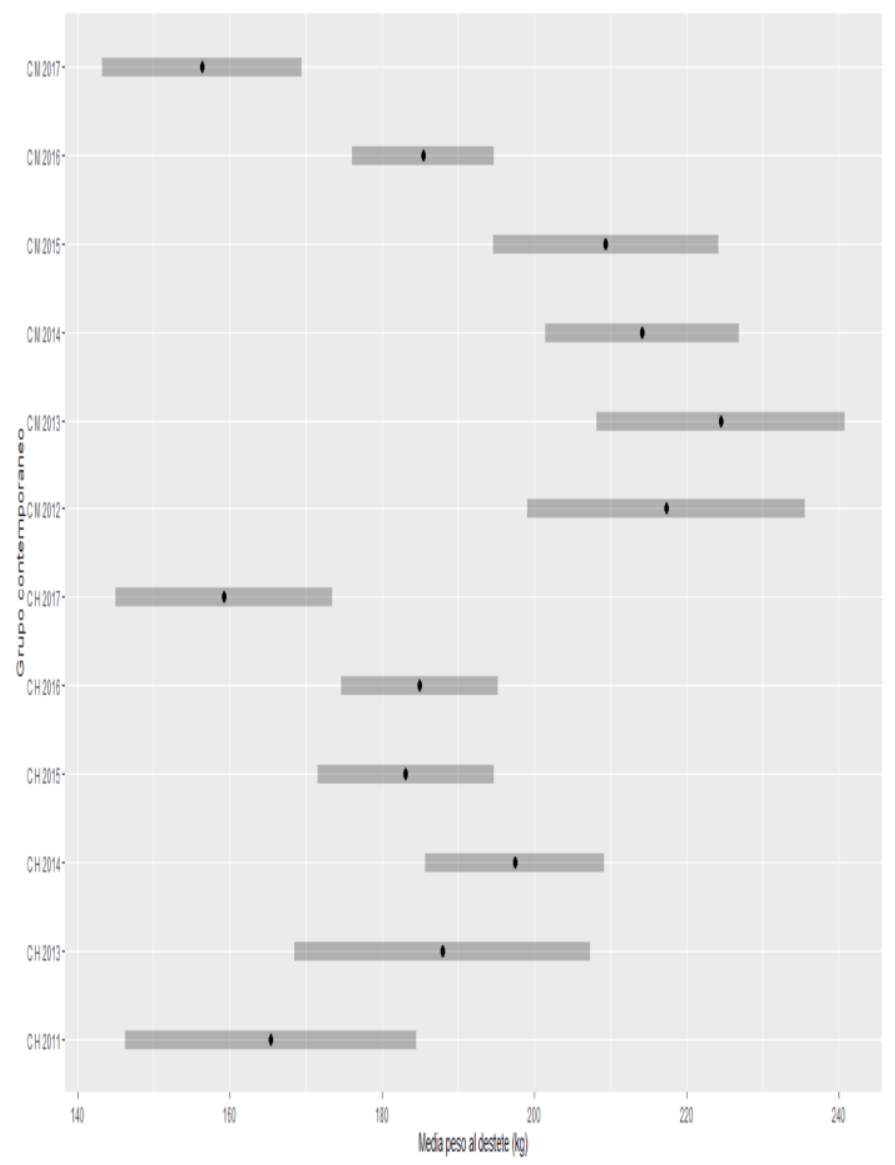

Fig.2. Interacción entre el año y sexo para el hato C.

Por otro lado, la Fig. 2 muestra la interacción entre el año y el sexo para el hato $\mathrm{C}$, se puede observar que para la mayoría de GC los machos presentaron mayor media de peso al destete para cada uno de los años evaluados. Sin embargo, el GC CH2017 (hembras pesadas en el año 2017 del hato C), presentó una mayor media para la característica PD en comparación con la media de los machos de este año. También, se puede observar, que para el caso de los machos hay una caída en la media de peso al destete a través de los años, más marcada que en las hembras. Esta situación puede evidenciar diferencias en manejo, en selección de animales, en las condiciones climáticas presentes en esos años, entre otras cosas; que permitió que las hembras del hato $\mathrm{C}$ presentaran una media de PD superior a los machos de su mismo hato.

Por otro lado, referente al número de parto se encontró que la menor media para P4M fue en los animales pertenecientes al primer y séptimo parto con un valor de $103.06 \mathrm{~kg}$ y $103.17 \mathrm{~kg}$, 
respectivamente; mientras que la mayor media se registró en animales de parto 3 con un valor de $119.55 \mathrm{~kg}$.

Para el caso del PD se encontró que el número de parto que en que se reportó menor media, fue de animales nacidos de primer parto y de siete o más partos con un valor de $191.30 \mathrm{~kg}$ y 206.09 $\mathrm{kg}$, respectivamente. A su vez, la mayor media de PD se reportó para animales nacidos del quinto parto con un valor de 213.06 $\mathrm{kg}$. En un estudio realizado en ganado criollo Romosinuano se encontró que las vacas de primer parto y las de cuatro o más partos, fueron las que destetaron terneros más livianos (184.70 $\mathrm{kg}$ y $179.56 \mathrm{~kg}$, respectivamente), mientras que las vacas de segundo y tercer parto destetaron terneros que no presentaron diferencias significativas en la media de los encontrándose valores de $185.26 \mathrm{~kg}$ y $185.65 \mathrm{~kg}$, respectivamente [4]. Para esta situación se puede observar que existe una superioridad de la raza $\mathrm{BON}$ con respecto a la raza Romosinuano del estudio mencionado anteriormente, para destetar terneros más pesados, ya que presenta para todos los partos aquí mencionados una mayor media de peso al destete.

En la GDP4M-D se encontró una media de $0,645 \mathrm{~kg}$ para animales nacidos de parto cuatro, siendo la mayor media obtenida entre todos los órdenes de parto en este estudio, en contraste, los números de parto uno y siete presentaron la menor media de GDP4M-D con valores de 0.581 y 0.556 , respectivamente. La habilidad de ganar peso en los terneros en esta etapa de la vida depende fuertemente de la habilidad materna de las vacas, debido a que es un periodo predestete [18].

La razón por la que existen diferencias entre las medias de $\mathrm{P} 4 \mathrm{M}$, PD y GDP4M-D en cada uno de los partos es porque las vacas presentan cambios morfológicos a lo largo de sus vidas que finalmente se ven reflejados en el crecimiento de sus crías. Por lo tanto, vacas que se encuentran en partos intermedios tienden a mostrar una habilidad materna mayor en comparación con vacas más viejas o novillas jóvenes, debido a que estas últimas destinan parte de los nutrientes consumidos para su desarrollo fisiológico y corporal y menos al proceso de lactación [21]. y por otro lado, las vacas de mayor edad (viejas), presentan un comportamiento lineal y cuadrático que implica que tengan promedios de pesos y ganancias de pesos menores. [22]

La época de pesaje suele ser otro efecto fijo que tiene influencia sobre algunas características de crecimiento; para este estudio se encontró que fue significativa para $\mathrm{PD}$, obteniéndose que terneros pesados en la época lluviosa uno (abril, mayo y junio) presentaron la mayor media de PD con un valor de $209.65 \mathrm{~kg}$ y los pesados en la época lluviosa dos (octubre, noviembre y diciembre) presentaron la menor media de PD de $196.69 \mathrm{~kg}$. De igual manera, la época resulto ser fuente de variación para la característica GDP4M-D. sin embargo, para ese caso la época involucrada fue la época de nacimiento de los animales y se encontró que en la época de lluvia uno (abril, mayo y junio) se presentó la mayor media de GDP4M-D con un valor de $0.64 \mathrm{~kg}$ y en la época de lluvia dos (octubre, noviembre y diciembre) tuvo animales BON con la menor media para GDP4M-D con un valor de $0.56 \mathrm{~kg}$. Esta situación puede deberse a que normalmente la época de lluvia uno, es la más fuerte en
Colombia y viene seguida de una sequía leve, lo que implica que haya más disponibilidad de forraje para los animales. Mientras que la época de lluvia dos, es menos intensa, por lo que es probable que la disponibilidad de forraje sea menor. Cabe aclarar que los efectos de la lluvia no se ven inmediatamente y quizás por esta razón no se ven las medias de GDP4M.D más bajas en esta época.

Es importante tener en cuenta que tanto los datos de peso (P4M o PD) como la ganancia de peso (GDP4M-D), se obtiene cuando estos animales son pesados, viene influenciado por las condiciones ambientales, de disponibilidad de forraje en cuanto a calidad y cantidad, entre otras circunstancias que ocurren a lo largo de la vida de estos animales.

\section{CONCLUSIONES}

El mejor modelo lineal para las características de crecimiento pre-destete evaluadas en este trabajo fue aquel que incluyó el GC, mostrando mejores valores de AIC, SCE y $\mathrm{R}^{2}$.

La edad como efecto fijo para la característica P4M y la edad como covariable para PD permitieron obtener mejores valores de AIC, BIC, SCE y $\mathrm{R}^{2}$.

El número de variables que son incluidas en un modelo lineal tienen influencia en el valor del $\mathrm{R}^{2}$.

Las variables que resultaron tener efecto significativo fueron, número de parto, grupo contemporáneo y edad para el caso de $\mathrm{P} 4 \mathrm{M}$ y $\mathrm{PD}$, sugiriendo que tienen influencia en el crecimiento pre-destete de la raza BON.

\section{AGRADECIMIENTO}

A los propietarios de los hatos que permitieron el uso de sus datos y al Colciencias por financiar el proyecto "Conociendo nuestros recursos criollos: análisis genómico y búsqueda de regiones del genoma asociadas a características productivas, reproductivas y de salud en ganado Blanco Orejinegro", con código de Colciencias 110177658049 y número de contrato P44842-015-2018

\section{REFERENCIAS}

[1] G. Bedoya, L. G. Carvajal, F. L. Moreno, S. Davies, J. Derr, J. E. Ossa, y, U. Kingdom, "Estructura molecular y poblacional del ganado criollo Colombiano (GCC)". Vol. 14, no.2, págs. 109-120, 2001.

[2] A. López Herrera, O. Saldarriaga, A. Arango, M. Rugeles López, F. Zuluaga Tobón, M. Olivera Ángel, N. Bermúdez, G. Bedoya, y J. Ossa Londoño, "Ganado Blanco Orejinegro (BON): Una alternativa para la producción en Colombia", Revista Colombiana de Ciencias Pecuarias, vol. 14, no. 2, págs.121-128, Agosto de 2001.

[3] G. M. Correal, , "El Ganado Criollo Colombiano Blanco Orejinegro (Bon)". Animal Genetic Resources Information, vol. 9, págs. 27-

35, abril $\quad$ de 1992. DOI: https://doi.org/10.1017/S1014233900003175 
[4] G. A. Ossa, M. A. Suárez, y J. E. Pérez, "Efectos del medio y la herencia sobre los pesos al nacimiento, al destete y a los 16 meses de edad en terneros de la raza criolla Romosinuano". Corpoica Ciencia y Tecnología Agropecuaria, vol. 8, no. 2, págs. 81-92, 2008, DOI: 10.21930/rcta.vol8_num2_art:98

[5] M. Poveda, y C. Gutierrez, "Evaluación de ganancia de peso de terneros cebú sometidos a tres tratamientos antihelminticos en un hato de cría comercial de Cumaral (Meta)", Bogotá, Colombia, enero de 2016.

[6] J. C. Quintero, J. G . Triana, J. H. Quijano, y E. Arboleda, "Influencia de la inclusión del efecto materno en la estimación de parámetros genéticos del peso al destete en un hato de ganado de carne", vol. 20, págs. 117-123, 2007.

[7] R Core Team, "A Language and Environment for Statistical Computing. R Foundation for Statistical Computing", https://www.r-project.org, 2018.

[8] J. C. Souza, L. O. C. Silva, A. Gondo, J. A. Freitas, C. H. M. Malhado, F. P. B. Ferrar, J. R. B. Sereno, R. L. Weaber, y W. R. Lamberson, "Parâmetros e tendências genéticas do peso de bovinos criados á pasto no brasil", Archivos de Zootecnia, vol. 60, no. 231, págs. 457-465. 2011. DOI: 10.21071/az.v60i231.4504

[9] C. Pereira, C. U. Faria, y R. Lôbo, "A importância da qualidade da informação na predição de valores genéticos para características de crescimento em bovinos da raça Nelore", Arquivo Brasileiro de Medicina Veterinaria e Zootecnia, vol. 69, no. 2, págs. 465-473, 2017. DOI: $10.1590 / 1678-4162-8942$.

[10] T. S. De Lira, L. de S. Pereira, F. B. Lopes, J. L. Ferreira, R. B. Lôbo y G. C. de J. Santos, "Tendências genéticas para características de crescimento em rebanhos nelore criados na região do trópico úmido do Brasil”. Ciencia Animal Brasileira, vol. 14, no.2, págs. 23-31, junio de 2013. DOI: 10.5216/cab.v14i1.16785

[11] A.V. Garnero, M.C.C.D. Muñoz, C.R. Marcondes, R.B. Lôbo, T. Lira, R.J. e Gunski, "parâmetros genéticos entre pesos pré e pósdesmama na raça nelore". Archivos de Zootecnia, vol. 59, no. 226, págs. 307-310, 2010.

[12] M. A. Revidatti, G. A. Crudeli, y C. J. Minoli, "Peso al nacimiento y evolución hasta el destete de terneros cruza Senepol vs. cruza Aberdeen Angus en Corrientes". Comunicaciones Científicas y Tecnológicas, págs. 1-3, 2000.

[13] G. Martínez, J. Torres, P. Guio, y H. Ballesteros, "Utilización de toros criollos y su efecto en rasgos de reproducción y crecimiento, en un sistema de cría en la Orinoquia colombiana". Actas Iberoamericanas de Conservación Animal, págs. 44-50. 2015.

[14] R. Martínez, R. E. Vásquez, y J. L. Gallego, "Eficiencia productiva de la raza BON en el trópico colombiano". In Journal of Visual Languages \& Computing, Vol. 1, 2012. DOI: http://hdl.handle.net/20.500.12324/13048

[15] J. Cañas, J. Ramirez, O. Arboleda, J. Ochoa, O. Vergara y M. Cerón." Peso al destete en ganado Blanco Orejinegro (BON) en el noroccidente colombiano", Rev. MVZ Córdoba, vol.13, no.1, págs. 1138-1145, 2008. DOI: https://doi.org/10.21897/rmvz.405

[16] E.J. Ramírez Toro, R. J. Ocampo Gallego, W.O. Burgos Paz, M. A. Elzo, R. A. Martínez Sarmiento, y Cerón Muñoz M. F, "Estimación poligénica y genómico-poligénica para características de crecimiento en ganado Blanco Orejinegro (BON)". Livestock Research for Rural Development. Vol. 31, no. 3, 2019. DOI: http://www.lrrd.org/lrrd31/3/ceron31030.html

[17] A. Galeano, "Evaluación Genética del recurso animal de los sistemas de producción de bovinos en doble propósito en Colombia". In Universidad Nacional de Colombia, Bogotá, Colombia, junio de 2010.
[18] J. C. D. C. Paneto, D. C. Lemos, L. A. F. Bezerra, R. Martins Filho, y R. B. Lôbo, "Estudo de características quantitativas de crescimento dos 120 aos 550 dias de idade em gado Nelore", Revista Brasileira de Zootecnia, vol. 31, no. 2, págs. 668-674, 2002. DOI: https://doi.org/10.1590/S1516-35982002000300017

[19] J. Dominguez, F. Rodriguez, J. Ortega y A. Flores. "Selección de modelos, parámetros genéticos y tendencias genéticas en las evaluaciones genéticas nacionales de bovinos Brangus y Salers". Agrociencia, vol.43, no.2, págs. 107-117. febrero de 2009.

[20] E. Martínez, "Errores frecuentes en la interpretación del coeficiente de determinación lineal". Anuario Jurídico y Económico Escurialense, vol. 38, págs. 315-332, 2005.

[21] F. Molina, A.Gómez, J. Guerrero, A. Garrido, J. Adame, y D. Pérez, "Efecto de varios factores en el peso al destete de los terneros en una explotación comercial", Jornadas Sobre Producción Animal, vol. 32, págs. 30-32, 2017.

[22] G. De Jesus Santos, T. Santos de lira, L.de Souza Pereira, F. Brito, J. Ferreira. "Efeitos não genéticos sobre características produtivas em rebanhos nelore criados na região norte do Brasil". Acta veterinaria Brasilica, vol.5, págs. 385-392, 2011. DOI: https://doi.org/10.21708/avb.2011.5.4.2356

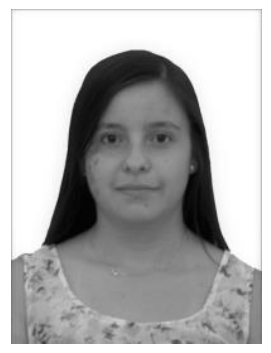

Luisa F Naranjo Guerrero. Nació en Bogotá D.C. Colombia, en 1995. Recibió el título de zootecnista de la Universidad Nacional de Colombia en 2019. De 2018 a 2019 fue integrante del grupo de investigación de Biodiversidad y Genética Molecular, BIOGEM, de la Universidad Nacional de Colombia. Realizó su trabajo de grado en el área de mejoramiento genético animal, bajo un macroproyecto financiado por COLCIENCIAS.

ORCID: https://orcid.org/0000-0003-0743-0655

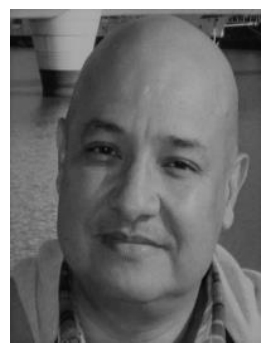

Albeiro López Herrera. Nació en Bello (Antioquia), Colombia, en 1966. Recibió el título de Zootecnista de la Universidad Nacional de Colombia, Sede Medellín en 1990 y de Médico Veterinario de la Universidad de Antioquia en 1995. Entre 1998 y 2000 realizó su maestría en Ciencias Básicas Biomédicas énfasis Virología y luego su Doctorado entre 2000 y 2004 en Ciencias Biomédicas énfasis Virología, ambos en el tema de resistencia Natural del Ganado BON a infecciones virales, en la Corporación de Ciencias Básicas Biomédicas de la Universidad de Antioquia, entre 2004 y 2005 realizó un posdoctorado en Neuro sida en IUPUI (Indiana University Purdue University Indianapolis). A partir del 2005 está vinculado al Departamento de Producción Animal de la Facultad de Ciencias Agrarias de la Universidad Nacional de Colombia, sede Medellín, en la categoría de Profesor Titular desde el 2016. Actualmente es director del grupo de investigación de Biodiversidad y Genética Molecular, BIOGEM, de la Universidad Nacional de Colombia y es 
reconocido como Investigador Senior por el Ministerio de Ciencia, Tecnología e innovación (Minciencias).

ORCID: https://orcid.org/0000-0003-1444-3470

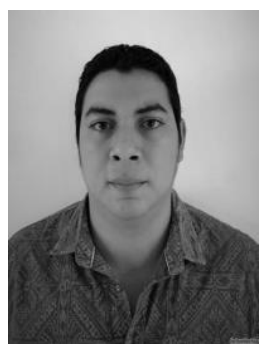

Juan Carlos Rincón Flórez. Nació en Medellín (Antioquia), Colombia, en 1986. Recibió el título de Zootecnista de la Universidad Nacional de Colombia, Sede Medellín en 2009. Entre 2010 y 2011 realizó su maestría en Ciencias agrarias con énfasis en genética y mejoramiento animal y entre 2012 y 2015 un doctorado en Ciencias Agrarias con énfasis en genética molecular y mejoramiento animal de la Universidad Nacional de Colombia. Es profesor Asociado de la Universidad Nacional de Colombia, sede Palmira, en el Departamento de Ciencia Animal de la Facultad de Ciencias Agropecuarias. Actualmente hace parte del grupo de investigación de Biodiversidad y Genética Molecular, BIOGEM, de la Universidad Nacional de Colombia y es reconocido como Investigador Asociado por el Ministerio de Ciencia, Tecnología e innovación (Minciencias).

ORCID: https://orcid.org/0000-0002-6769-6407

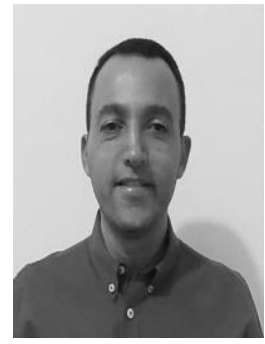

Luis Gabriel González-Herrera. Nació en Manizales (Caldas) Colombia, en 1978. Recibió el título de Médico Veterinario Zootecnista de la Universidad de Caldas en 2004. Entre 2004 a 2006 realizó su maestría en Genética y Mejoramiento Animal y entre 2009 y 2013 su doctorado en Genética y Mejoramiento Animal, ambos en la Universidade Estadual

Paulista, Sao Paulo, Brasil. Es profesor Asociado de la Universidad Nacional, sede Medellín, en el Departamento de Producción Animal de la Facultad de Ciencias Agrarias y hace parte del grupo de investigación de Biodiversidad y Genética Molecular, BIOGEM, de la Universidad Nacional de Colombia. Reconocido como Investigador Senior por el Ministerio de Ciencia, Tecnología e innovación (Minciencias).

ORCID: https://orcid.org/0000-0003-1444-3470 